Article title: Transformative Research Paradigm: A Response to SDG 4 by Intensifying Support Scholarships for Deaf and Hard-of-Hearing Students at an Open Distance and E-Learning University

Authors: Tonny Matjila[1], Petro van der Merwe[2]

Affiliations: University of South Africa[1]

Orcid ids: 0000-0002-6583-7085[1], 0000-0002-1330-9205[2]

Contact e-mail: tmatjit@unisa.ac.za

License information: This work has been published open access under Creative Commons Attribution License http://creativecommons.org/licenses/by/4.0/, which permits unrestricted use, distribution, and reproduction in any medium, provided the original work is properly cited. Conditions, terms of use and publishing policy can be found at https://www.scienceopen.com/.

Preprint statement: This article is a preprint and has not been peer-reviewed, under consideration and submitted to UnisaRxiv for open peer review.

Funder: N/A

DOI: $10.25159 /$ UnisaRxiv/000010.v1

Preprint first posted online: 07 March 2021

Keywords: Deaf, hard of hearing, open distance and e-learning , student support, transformative research paradigm, transformative mixed-method research 


\section{Transformative Research Paradigm: A Response to SDG 4 by Intensifying Support Scholarships for Deaf and Hard-of-Hearing Students at an Open Distance and E-Learning University}

\section{Tonny Nelson Matjila}

https://orcid.org/0000-0002-6583-7085

University of South Africa

tmatjit@unisa.ac.za

\author{
Petro van der Merwe \\ https://orcid.org/0000-0002-1330-9205 \\ University of South Africa
}

\section{Abstract}

The landscape of higher education in South Africa is beset with numerous challenges including accommodating students who are Deaf and hard of hearing. The reasonable accommodation in place does not seem to redress the daily challenges faced by these students at an open distance e-learning university despite the policies and legislature in place. The Department of Higher Education and Training in South Africa, through its initiatives such as the Strategic Disability Policy Framework on Disability for the Post-School Education and Training System, aims to fast track and respond to Sustainable Developmental Goal 4. This paper argues how the transformative research paradigm may be a response to this mandate. Firstly, the paradigm suggests theories such as the critical disability theory and the transactional distance theory and discusses their relevance in promoting scholarships for disability in open distance e-learning. Secondly, it highlights the transformative assumptions on ontology, epistemology, axiology, and a methodology which are often ignored when developing interventions on disability issues. The ontology helps to understand the reality through the lenses of students who are Deaf and hard of hearing, epistemology deals with acquiring knowledge of the subjects and literature, and axiology deals with the ethical considerations for the said cohort. The transformative mixed-method research deals with the inequalities and makes provisions to include, deal with, and guarantee the integration of qualitative and quantitative data sets.

Keywords: deaf, hard of hearing, open distance and e-learning, student support, transformative research paradigm, transformative mixed-method research

\section{Introduction}

There seems to be increased access for students who are Deaf and hard of hearing (SDHH) to study at an open distance e-learning (ODeL) university in South Africa. This has come with several inclusion, integrational and transformational challenges despite the legislature and policies in place. Ntombela $(2020,18)$ highlights the perspectives of the inclusion challenges and the lack of student support scholarships in this field which 
are critical to accelerating the response to the South African National Development Plan (NDP) 2030, which in turn feeds into the United Nations Sustainable Development Goals (SDGs), specifically goal number four with a focus on two targets (UN 2021). Firstly, target 4.5 speaks to eliminating gender disparities in education and ensuring equal access to all levels of education and to vocational training for the vulnerable, including persons with disabilities, indigenous peoples, and children in vulnerable situations. Secondly, target 4. b responds to expanding scholarships in Education on disability studies of which the contribution is conversely proportional to target 4.5 .

The Department of Higher Education and Training (DHET) in South Africa has developed a Strategic Disability Policy Framework on Disability for the Post-School Education and Training System which is intended to monitor the development of structured educational models and programmes for students with disabilities in higher education (DHET 2018, 75-83). From this strategic framework, models of disability are fundamental to understand and to deal with reasonable accommodation for SDHH in ODeL.

Mertens (2015, 3-6) asserts that the transformative research paradigm may be a panacea provided the philosophical foundations and assumptions are dealt with through ontology (the reality of barriers faced by SDHH in ODeL), epistemology (the way in which knowledge production should be realised), axiology (ethical principles and considerations when approaching matters of deafness and labelling thereof), and methodology (how to do obtain findings about the reality and reliable answers while applying scientific methods).

\section{Transformative Underpinnings and Perspectives}

Considering the foregoing highlighted developments, this paper seeks to initiate discussion about the provision of student support in ODeL and the level of inclusion in line with SDG 4. Theories are one of the major pillars of research that influences, advances and transforms epistemological position in various disciplines (Ukwoma and Ngulube 2021, 48). A theoretical framework through the identified and applied theories thus gives a reflection on support scholarships for SDHH at an ODeL university. Varpio et al. (2020, 989), and Grant and Osanloo (2014) refer to the theoretical framework as a blueprint for an imperial study.

Alavi et al. $(2018,528)$ provide guidelines for the way in which the theoretical framework and research methods may be linked through the research paradigm. Failure to link these may result in a faulty study in which the research issue may be inconsistent with the methodology. From these guidelines, one can infer that it is important to identify the theoretical orientation that will not only promote comprehension but should also direct the design of research in which dynamic phenomena such as hearing impairment are at the core of the investigation. 
In the scope of the transformative research paradigm focusing on SDHH, the selection of the theories to be applied provided a coherent explanation in line with the focus on the provision of support to SDHH in ODeL. Similarly, there was a conceptual basis for understanding, evaluating, and constructing ways of exploring the research problem; the critical disability theory (CDT) and the transactional distance theory accommodated this context (Moore and Mertens 2015, 147).

\section{Critical Disability Theory}

Rembis $(2019,377)$ advocates the view that the literature on the marginalisation of minorities in societies is receiving considerable attention. The transformative paradigm emerged as a result to redress the shortcomings of the two dominant paradigms, namely, the positivist paradigm and the constructivism paradigm (Mertens 2010,9). This view is corroborated by Hall $(2019,3)$ who argues that CDT applies to a theoretical approach involving diversity and interdisciplinarity. Hall also aligns CDT with the transformative research paradigm that promotes activism and confines insights not only to academia but also to the broader public. There appears to be a consensus among the researchers on the purposes and goals of CDT, but some researchers such as Schalk $(2017,2)$ places CDT as a methodology rather than as a research framework, applying the same purposes as others.

Consequently, CDT responds to the transformative research paradigm in which the researcher interacts with the participants with central attention of the research effort on discovering agency, concealed by social practices, which contribute to liberation and emancipation. Secondly, it provides a coherent explanation of the concepts, Deaf and hard of hearing, through the models of disability. It also provides guidelines for the way in which the Universal Design for Learning Framework can be incorporated into the disability ideological stance through a debate about the continuum of the two dominant models of disability, namely, the clinical-pathological model known as the medical model and the social-cultural model known as the social model of disability (Kivunja and Kuyini 2017, 38). The consideration of this theory through the Universal Design for Learning eliminates parallel support structures on the provision of student support and has one system. This system accommodates all in line with principles that guide the design of inclusive instruction in the classroom and accessible courses in line with the definition by the Center for Applied Special Technology (2011):

- numerous representation methods give learners a variety of ways to gain information and build knowledge;

- numerous means of student action and expression provide alternatives for learners to demonstrate what they have learnt; and

- numerous ways of engaging students tap into the interests of learners, challenge them appropriately and motivate them to learn. 


\section{Transactional Distance Theory}

The context is crucial to research and the transactional distance theory affords that and gives guidelines for the way in which to support SDHH fully at an ODeL setting (Bozkurt 2019, 53). Pitsoe and Letseka $(2016,83)$ concur with the functions and aims of student support as proposed by Tait $(2000,289)$ while dealing with the need to care for all students irrespective of gender, disability or any other factor. These functions are in line with the theoretical framework which provided the structure for defining the way in which (philosophically, epistemologically, methodologically, and analytically) the study has been approached to promote interdisciplinarity scholarships for student support in ODeL in the context of deafness and hard of hearing across various disciplines such as Psychology, Distance Learning Education, Disability Studies, and Inclusive Education. These functions deal with the following comprehensive components of student support:

- Cognitive: Supporting and improving learning through the mediation of the normative and standardised aspects of individual students' course materials and learning tools.

- Affective: Providing an atmosphere that nurtures students, creates dedication and increases self-esteem.

- Systems: Establishing reliable, accessible, and usually student-friendly administrative processes and information management systems.

\section{Transformative Assumptions}

The transformative paradigm that focuses on empowering and eradicating despotic formations around research participants concerning student support functions is supported by Pitsoe and Letseka $(2016,83)$. These functions include reasonable accommodations for South African Sign Language (SASL), which is often the first language of most deaf students. As guided by Ivankova and Wingo $(2018,987)$, this paradigm based its work on social justice problems and aims to tackle the social, economic and political concerns that contribute to societal inequality, confrontation, resistance and power dynamics at all levels (Cram and Mertens 2016, 163).

The critics of this paradigm warn that emancipation and transformation may not be achieved and are not guaranteed as the improvement on the provision of support for the SDHH may be marginal or non-existent. Also, improvement may not be possible until the ODeL university becomes critically conscious of this exclusion and the impact it has on attrition and retention to even avoid the lives of certain participants be altered for the worse (Vehmas and Watson 2014, 644). This argument shows that participants are sometimes classified as belonging to a certain marginalised category, so homogeneous notions of identity are superimposed. Secondly, this paradigm does not recognise that different participants enter universities to study with different levels of 
awareness and layers, and disability diversity. It naively assumes that societies are blindly bidding for powerful regimes, enslaving themselves more in the process.

Drawing from Lincoln and Guba $(1988,7)$ and Mertens $(2015,5)$, this paradigm considers the axiology and values of respect to counteract the alluded argument. Building rapport with the participants is therefore key to eliminate the ontological assumptions and understanding the layers of deafness and hard of hearing (Kivunja and Kuyini 2017, 29, 38). This is often considered a progressive model as it assumes a transactional epistemology in which the researcher communicates with the participants, an ontology of historical reality, particularly when it relates to exploitation, and a dialogic methodology and axiology that respect cultural norms, when it seeks to shift policies, tackle systemic injustice, and promote social equity (McIntyre-Mills and Romm 2019, 465).

\section{Ontology}

Cultural relativism is often rejected when promoting and recognising different versions of what constitutes reality from the participants. Widianingsih and Mertens $(2019,28)$ link transformative research to SDG 4 by explaining the need to consider that privilege is granted to different versions of reality over others and that it is important to objectively analyse the privileged views to assess what is lacking when the views of marginalised people are not privileged. The marginalised people in the context of this study are the SDHH at an ODeL university; it was important to consider all versions of their reality and layers of disability including deafness and hard of hearing whenever the system is evaluated or policies are reviewed. The central ontological assumption question to be explored is: What version of truth offers an awareness that can lead to improvements in the status quo which in turn can lead to social justice being promoted?

One of the assumptions highlighted in the literature is that institutions of higher education often assume that any SASL interpreter can translate anything. However, translators should be familiar with the module content as is the case with every translator who should be familiar with the linguistic aspects of the language they are translating.

\section{Epistemology}

The transformative epistemological assumptions pose concerns about the existence of a researcher who may be a non-member of the researched marginalised community, meaning a researcher who may not be Deaf or hard of hearing while conducting a study on this topic. Mertens $(2012,807)$ advises that this may be managed and dealt with by building a rapport with the community who in this context may include SDHH and Sign language interpreters among other stakeholders. 
Axiology

Cram and Mertens $(2016,164)$ discuss the transformative axiological assumptions that include the cultural histories, norms and identities. These are critical when promoting social justice and being cognisant of the discrimination related to labels and other oppressive factors. Cultural competence is, therefore, a vital provision connected to the researcher's ability in culturally diverse cultures to accurately reflect the reality on the ground. This is supported by Romm $(2018,471)$ who emphasises the importance of cultural competence and the way in which it should form an integral part of the principle for those operating within the philosophical assumptions of the transformative framework.

\section{Methodology}

Transformative mixed-method research (TMMR) is the contemporary approach to social analysis that has taken centre stage in recent debates about research methodology (Provinzano, Sondergeld, and Knaggs 2020, 3). In addition to offering more accurate, reliable and richer outcomes and inferences relative to standard mono-method research, TMMR was suggested and favoured as a response to the paradigm wars (Chih-Pei and Chang 2017, 206). TMMR consists of mixed methods that redress the inequities and that are informed by the outlined transformative assumptions (Camacho 2020, 309). The transformative methodological assumptions flow from the three foregoing assumptions. It must consider the axiological assumptions and incorporate the Sign Language Community Terms of Reference for research with people who are Deaf and hard of hearing (Fan 2019, 41; Harris, Holmes, and Mertens 2009, 114).

Similarly to mixed-methods research (MMR), TMMR guarantees the integration of both the quantitative and qualitative approaches through the sequential approach and strategies. This integration through quantitative and qualitative data, synthesises and then translates to richer evaluation the inference for evaluation practitioners (Gibson 2017, 199). Therefore, the use of a mono approach for evaluating the existing student support framework at an ODeL university would have limited the study to achieve its objectives. Consequently, and in line with the rationale for MMR outlined by Shorten and Smith $(2017,3)$, the application of TMMR should be appropriate for exploring the outlined research questions.

Therefore, TMMR becomes appropriate owing to its ability to complement quantitative and qualitative approaches based on their strengths and limitations. At the practical level, it allows and makes a provision for the use of both qualitative and quantitative databases to solve a complex societal problem by not diverting from the transformative paradigm. At the procedural level, it helps us to develop a holistic understanding of the problem statement and how to solve it through the scientific process and procedures by having a deeper understanding of the changes that need to take place after evaluating the student support framework to reasonably accommodate SDHH in ODeL. It seems 
to be a useful strategy to understand the need for an intervention programme and its effects through the collection of both quantitative and qualitative data over time. Lastly, it allows the comparison of various positions taken from quantitative and qualitative data.

\section{Conclusion}

The student support scholarships in ODeL in the context of deafhood has a role to play in responding and contributing to the South African NDP 2030 and SDG 4. Furthermore, it is these scholarships that may translate to student support practices for SDHH. The transformative research paradigm seem to focus on philosophical foundations and assumptions which may lead to a more understanding and inclusive framework for conducting studies with and not for SDHH. Transferability as one pillar of trustworthiness of data may be realised for this study where ODeL universities may adopt the tenants of this paradigm while responding to the 2018 Strategic Disability Policy Framework on Disability for the Post-School Education and Training System by the DHET to deal with disability-related challenges in higher education. The transformative research paradigm gives researchers an opportunity to work with all stakeholders involved in the provision of student support including SDHH as part of knowledge production that may transform and emancipate employees at ODeL universities and the broader communities.

The CDT through the models of disability and Universal Design for Learning provides an understanding of applications that promote integration and inclusivity for reasonable accommodation for SDHH. The transactional distance theory further deals with the context of ODeL which is different to the traditional university structure to consider the cognitive, affective and systems functions of student support. The TMMR may contribute to unearthing relevant and valid epistemologies that may guide suitable pedagogies, heutagogies and andragogies to support SDHH in ODeL. These scholarships may accelerate the response to SDG 4 and pave the way for relevant policies and legislature for student support in ODeL.

\section{References}

Alavi, M., M. Archibald, R. McMaster, V. Lopez, and M. Cleary. 2018. "Aligning Theory and Methodology in Mixed Methods Research: Before Design Theoretical Placement." International Journal of Social Research Methodology 21 (5): 527-40. https://doi.org/10.1080/13645579.2018.1435016.

Bozkurt, A. 2019. "From Distance Education to Open and Distance Learning: A Holistic Evaluation of History, Definitions, and Theories." In Handbook of Research on Learning in the Age of Transhumanism, edited by S. Sisman-Ugur and G. Kurubacak, 252-273. Hershey: IGI Global. https://doi.org/10.4018/978-1-5225-8431-5.ch016. 
Camacho, S. 2020. "From Theory to Practice: Operationalizing Transformative Mixed Methods with and for the Studied Population." Journal of Mixed Methods Research 14 (3): 305-35. https://doi.org/10.1177/1558689819872614.

Center for Applied Special Technology. 2011. Universal Design for Learning (UDL) Guidelines: Full-Text Representation Version 2.0.

Chih-Pei, H. U., and Y. Y. Chang. 2017. "John W. Creswell, Research Design: Qualitative, Quantitative, and Mixed Methods Approaches." Journal of Social and Administrative Sciences 4 (2): 205-207.

Cram, F., and D. M. Mertens. 2016. "Negotiating Solidarity between Indigenous and Transformative Paradigms in Evaluation." Evaluation Matters - He Take Tō Te Aromatawai (New Zealand Council for Educational Research) 161-189. https://doi.org/10.18296/em.0015.

DHET (Department of Higher Education and Training). 2018. Strategic Disability Policy Framework on Disability for Post-School Education and Training System. Pretoria: Government Printing Works.

Fan, H. 2019. “An E-Learning Ecosystem for Deaf Young Adult Learners' English Literacy Attainment in India." PhD dissertation, University of Central Lancashire.

Gibson, C. B. 2017. "Elaboration, Generalization, Triangulation, and Interpretation: On Enhancing the Value of Mixed Method Research." Organizational Research Methods 20 (2): 193-223. https://doi.org/10.1177/1094428116639133.

Grant, C., and A. Osanloo. 2014. "Understanding, Selecting, and Integrating a Theoretical Framework in Dissertation Research: Creating the Blueprint for Your 'House'." Administrative Issues Journal Education Practice and Research 12-26. https://doi.org/10.5929/2014.4.2.9.

Hall, M. C. 2019. "Critical Disability Theory.” In Stanford Encyclopedia of Philosophy. https://stanford.library.sydney.edu.au/archives/win2019/entries/disability-critical/.

Harris, R., H. M. Holmes, and D. M. Mertens. 2009. "Research Ethics in Sign Language Communities." Sign Language Studies 9 (2): 104-31. https://doi.org/10.1353/sls.0.0011.

Ivankova, N., and N. Wingo. 2018. "Applying Mixed Methods in Action Research: Methodological Potentials and Advantages." American Behavioral Scientist 62 (7): 97897. https://doi.org/10.1177/0002764218772673.

Kivunja, C., and A. B. Kuyini. 2017. "Understanding and Applying Research Paradigms in Educational Contexts." International Journal of Higher Education 6 (5): 26-41. https://doi.org/10.5430/ijhe.v6n5p26.

Lincoln, Y. S., and E. G. Guba. 1988. "Criteria for Assessing Naturalistic Inquiries as Reports." 
McIntyre-Mills, J., and N. R. A. Romm. 2019. "Conclusion: Potential for Transformative Research to Address Risks.” In Democracy and Governance for Resourcing the Commons. Contemporary Systems Thinking, edited by J. McIntyre-Mills, N. Romm and Y. CorcoranNantes, 461-72. Cham: Springer. https://doi.org/10.1007/978-3-030-04891-4_16.

Mertens, D. M. 2010. "Philosophy in Mixed Methods Teaching: The Transformative Paradigm as Illustration." International Journal of Multiple Research Approaches 4: 9-18. https://doi.org/10.5172/mra.2010.4.1.009.

Mertens, D. M. 2012. “Transformative Mixed Methods: Addressing Inequities.” American Behavioral Scientist 6 (6): 802-13. https://doi.org/10.1177/0002764211433797.

Mertens, D. M. 2015. "Mixed Methods and Wicked Problems." Journal of Mixed Methods Research 3-6. https://doi.org/10.1177/1558689814562944.

Moore, E. A., and D. M. Mertens. 2015. "Deaf Culture and Youth Resilience in Diverse American Communities. Vol. 11.” In Youth Resilience and Culture. Cross-Cultural Advancements in Positive Psychology, edited by L. Theron, L. Liebenberg and M. Ungar, 143-55. Dordrecht: Springer. https://doi.org/10.1007/978-94-017-9415-2_11.

Ntombela, S. 2020. “Teaching and Learning Support for Students with Disabilities: Issues and Perspectives in Open Distance E-Learning." Turkish Online Journal of Distance Education 21 (3). https://doi.org/10.17718/tojde.761919.

Pitsoe, V., and M. M. Letseka. 2016. "Ubuntu Driven ODL Student Assessment.” In Open Distance Learning (ODL) through the Philosophy of Ubuntu, edited by M. Letseka, 93106. New York: Nova.

Provinzano, K. T., T. A. Sondergeld, and C. M. Knaggs. 2020. "Community Schools as a Sustainable Comprehensive School Reform Strategy: A Transformative Mixed Methods Perspective." Mid-Western Educational Researcher 32 (1): 3-30.

Rembis, M. 2019. "Challenging the Impairment/Disability Divide: Disability History and the Social Model of Disability." In Challenging the Impairment/Disability Divide, edited by N. Watson and S. Vehmas, 377-390. London: Routledge. https://doi.org/10.4324/9780429430817-27.

Romm, R. A. 2018. Responsible Research Practice: Revisiting Transformative Paradigm in Social Research. New York: Springer.

Schalk, S. 2017. "Critical Disability Studies as Methodology." Lateral: Journal of the Cultural Studies Association 61. https://csalateral.org/issue/6-1/forum-alt-humanities-criticaldisability-studies-methodology-schalk/.

Shorten, A., and J. Smith. 2017. "Mixed Methods Research: Expanding the Evidence Base." Evidence-Based Nursing 20 (3): 1-5. https://doi.org/10.1136/eb-2017-102699. 
Tait, A. 2000. "Planning Student Support for Open and Distance Learning." Open Learning: The Journal of Open, Distance and eLearning 287-99. https://doi.org/10.1080/713688410.

Ukwoma, S. C., and P. Ngulube. 2021. "Trends and Patterns of Theory Use in Open and Distance Education Research Journals 2009-2018." Open Learning. https://doi.org/10.1080/02680513.2021.1911793.

UN. 2021. "Sustainable Development Goal 4 (SDG 4)." In The 2030 Agenda for Sustainable Development. https://sdg4education2030.org/the-goal.

Varpio, L., E. Paradis, S. Uijtdehaage, and M. Young. 2020. “The Distinctions between Theory, Theoretical Framework, and Conceptual Framework." Journal of the Association of American Medical Colleges 95 (7): 989-994. https://doi.org/10.1097/ACM.0000000000003075.

Vehmas, S., and N. Watson. 2014. "Moral Wrongs, Disadvantages, and Disability: A Critique of Critical Disability Studies.” Disability and Society 29 (4): 638-50. https://doi.org/10.1080/09687599.2013.831751.

Widianingsih, I., and D. M. Mertens. 2019. “Transformative Research and the Sustainable Development Goals: Challenges and a Vision from Bandung, West Java." International Journal for Transformative Research 6 (1): 27-35. https://doi.org/10.1515/ijtr-2019-0005. 